\title{
Symmetric topological complexity of projective and lens spaces
}

\author{
JESÚS GONZÁLEZ \\ PETER LANDWEBER
}

\begin{abstract}
For real projective spaces, (a) the Euclidean immersion dimension, (b) the existence of axial maps and (c) the topological complexity are known to be three facets of the same problem. But when it comes to embedding dimension, the classical work of Berrick, Feder and Gitler leaves a small indeterminacy when trying to identify the existence of Euclidean embeddings of these manifolds with the existence of symmetric axial maps. As an alternative we show that the symmetrized version of (c) captures, in a sharp way, the embedding problem. Extensions to the case of even-torsion lens spaces and complex projective spaces are discussed.
\end{abstract}

$55 \mathrm{M} 30,57 \mathrm{R} 40$

Dedicated to the memory of Bob Stong

\section{Main result}

The Euclidean immersion and embedding questions for projective spaces were topics of intense research during the beginning of the second half of the last century. In the case of real projective spaces, the immersion problem has recently received a fresh push, partly in view of a surprising reformulation in terms of a basic concept arising in robotics, namely, the motion planning problem of mechanical systems. In more detail, Farber, Tabachnikov and Yuzvinsky [14] showed that for $r \neq 1,3,7$, the immersion dimension of $\mathrm{P}^{r}$-the $r$-dimensional real projective space-agrees with $\mathrm{TC}\left(\mathrm{P}^{r}\right)-1$, one unit less than the topological complexity of $\mathrm{P}^{r}$ (see Definition 1.1 and Theorem 4.2 below). In this paper we accomplish a completely analogous goal by connecting the Euclidean embedding dimension of $\mathrm{P}^{r}$ with Farber-Grant's notion of symmetric motion planning. Before stating our main results, we recall the relevant definitions.

The Schwarz genus [24] of a fibration $p: E \rightarrow B$, denoted by genus $(p)$, is the smallest number of open sets $U$ covering $B$ in such a way that $p$ admits a (continuous) section over each $U$. 
Definition 1.1 The topological complexity of a space $X, \operatorname{TC}(X)$, is defined as the genus of the endpoints evaluation map ev: $P(X) \rightarrow X \times X$, where $P(X)$ is the free path space $X^{[0,1]}$ with the compact-open topology.

TC $(X)$ is a homotopy invariant of $X$. Thinking of $X$ as the space of configurations of a given mechanical system, $\operatorname{TC}(X)$ gives a measure of the topological instabilities in a motion planning algorithm for $X$-a perhaps discontinuous (but global) section of the map ev. We refer the reader to Farber [10] for a very useful survey of results in this area, and to the Farber's book [11] for a thorough introduction to the new mathematical discipline of topological robotics.

We now come to the main definition (introduced and explored by Farber and Grant [12]). For a topological space $X$, let $\Delta_{X}$ be the diagonal in $X \times X$ and $\mathrm{ev}_{1}: P_{1}(X) \rightarrow$ $X \times X-\Delta_{X}$ be the restriction of the fibration ev in Definition 1.1. Thus $P_{1}(X)$ is the subspace of $P(X)$ consisting of paths $\gamma:[0,1] \rightarrow X$ with $\gamma(0) \neq \gamma(1)$. Note that ev 1 is a $\mathbb{Z} / 2$-equivariant map, where $\mathbb{Z} / 2$ acts freely on both $P_{1}(X)$ and $X \times X-\Delta_{X}$, by running a path backwards in the former, and by switching coordinates in the latter. Let $P_{2}(X)$ and $B(X, 2)$ denote the corresponding orbit spaces, and let $\mathrm{ev}_{2}: P_{2}(X) \rightarrow$ $B(X, 2)$ denote the resulting fibration.

Definition 1.2 With the above conditions, the symmetric topological complexity of $X$, $\mathrm{TC}^{S}(X)$, is defined by $\mathrm{TC}^{S}(X)=\operatorname{genus}\left(\mathrm{ev}_{2}\right)+1$.

Finally, let $E(r)$ stand for the Euclidean embedding dimension of $\mathrm{P}^{r}$. Then, our main result is:

Theorem 1.3 For $r>15$ as well as for $r \in\{1,2,4,8,9,13\}$, the symmetric topological complexity of $\mathrm{P}^{r}$ satisfies $\mathrm{TC}^{S}\left(\mathrm{P}^{r}\right)=E(r)+1$.

Before introducing an alternative characterization of $\mathrm{TC}^{S}\left(\mathrm{P}^{r}\right)$ (which implies Theorem 1.3), it is convenient to say a few words (to be expanded in Section 4) comparing the immersion and the embedding situations. It is known that the key concept bridging the immersion dimension of $\mathrm{P}^{r}$ to its topological complexity is that of an axial map. Not only do axial maps capture, in a sharp way, the immersion problem for projective spaces (see Adem, Gitler and James [1]), but as shown by Farber, Tabachnikov and Yuzvinsky [14], they conveniently encode instructions for the motion planning problem associated to $\mathrm{TC}\left(\mathrm{P}^{r}\right)$. Now, the work of Berrick, Feder and Gitler [4] does show a relation, at least in Haefliger's metastable range, between embeddings of real projective spaces on the one hand and symmetric axial maps on the other. However, as of today, this relation has an unsettled indeterminacy of one dimension-spelled out 
in (14) below. Instead, motivated by the main trick in [4] (see the proof of Proposition 2.2), our approach leads to a direct proof of Theorem 1.3. To this end we actually need to give up using the concept of symmetric axial map, and replace it by that of the level of an involution (as defined in (1) below). This allows us to get the following sharp and unrestricted characterization for the symmetric topological complexity of $\mathrm{P}^{r}$.

\section{Theorem 1.4 For all values of $r, \mathrm{TC}^{S}\left(\mathrm{P}^{r}\right)=\operatorname{level}\left(\mathrm{P}^{r} \times \mathrm{P}^{r}-\Delta_{\mathrm{P}^{r}}, \mathbb{Z} / 2\right)+1$.}

Here the pair $\left(\mathrm{P}^{r} \times \mathrm{P}^{r}-\Delta_{\mathrm{P}^{r}}, \mathbb{Z} / 2\right)$ stands for the $\mathbb{Z} / 2$-action on $\mathrm{P}^{r} \times \mathrm{P}^{r}-\Delta_{\mathrm{P}} r$ that interchanges coordinates, whereas the level of a principal $\mathbb{Z} / 2$-action on a space $X$, level $(X, \mathbb{Z} / 2)$, is defined by the formula

$$
\operatorname{level}(X, \mathbb{Z} / 2)=\min \left\{\ell>0: \exists \mathbb{Z} / 2 \text {-equivariant map } X \rightarrow S^{\ell-1}\right\}
$$

where the sphere is considered with the antipodal $\mathbb{Z} / 2$-action (see Dai and Lam [5]).

The paper is organized as follows. Section 2 is devoted to the proof of Theorem 1.4. After observing that Theorem 1.3 is a consequence of Theorem 1.4 together with Haefliger's characterization of Euclidean embeddings of smooth manifolds, in Section 3 we make an ad hoc analysis of the numerical values of $\mathrm{TC}^{S}\left(\mathrm{P}^{r}\right)$ for those cases of $r$ outside Haefliger's metastable range. Section 4 surveys the relation of axial maps to immersion dimension [1], and to (not necessarily symmetric) topological complexity [14], focusing on the way those ideas compare to (and motivate) our results. In Section 5 we study the symmetric topological complexity of $m$-torsion lens spaces, for $m$ even. Here our results are weaker than the case $m=2$, due in part to the fact that, as the 2-torsion increases, the end terms in (15) below start measuring different phenomena, thus preventing us from closing the cycle of inequalities. Yet, we manage to give alternative characterizations (Theorem 5.3 and Proposition 5.5) for the symmetric topological complexity of even-torsion lens spaces. One of these characterizations leads to a particularly convenient upper bound (Corollary 5.4) which depends not only on the dimension of the lens space, but also on its torsion. Theorem 5.8 illustrates the use of such an upper bound in the context of nonsymmetric topological complexity. In the final Section 6 we compute the numerical value of the symmetric topological complexity of complex projective spaces.

Acknowledgements The first author gratefully acknowledges the kind support received from Professor Michael Farber and the Department of Mathematical Sciences at Durham University during a visit in October 2008. Michael Farber's suggestions to an earlier version of this work were very helpful. In particular, Farber noticed that our proof of Theorem 1.3 actually leads to the proof of Theorem 1.4 and that our results and methods in Section 5 (originally written for $2^{e}$-torsion) apply just as well 
to even-torsion lens spaces. Helpful suggestions of an anonymous referee to an earlier version of this work led to a substantial improvement in the organization of the paper.

\section{Main proof}

There are three ingredients in the proof of Theorem 1.4. For the first one we note that Corollary 1 on page 97 of Schwarz [24] affirms that the canonical $\mathbb{Z} / 2$-cover $S^{n-1} \rightarrow \mathrm{P}^{n-1}$ classifies $\mathbb{Z} / 2$-covers of genus at most $n$. Explicitly, the principal $\mathbb{Z} / 2$-actions on a space $X$ which admit a $\mathbb{Z} / 2$-equivariant map $X \rightarrow S^{n-1}$ are precisely those for which the canonical projection $p: X \rightarrow X /(\mathbb{Z} / 2)$ has genus $\leq n$. In particular,

$$
\operatorname{genus}(p)=\operatorname{level}(X, \mathbb{Z} / 2) \text {. }
$$

Propositions 2.1 and 2.2 below are the other two auxiliary ingredients. They are based on the following preliminary constructions. For a path $\gamma \in P\left(\mathrm{P}^{r}\right)$, let $\hat{\gamma}:[0,1] \rightarrow S^{r}$ be any lifting of $\gamma$ through the canonical projection $S^{r} \rightarrow \mathrm{P}^{r}$, and then set $f(\gamma)$ to be the class of $(\hat{\gamma}(0), \hat{\gamma}(1))$ in the Borel construction $S^{r} \times_{\mathbb{Z} / 2} S^{r}=\left(S^{r} \times S^{r}\right) /(-x, y) \sim(x,-y)$. This gives a $\mathbb{Z} / 2$-equivariant commutative diagram

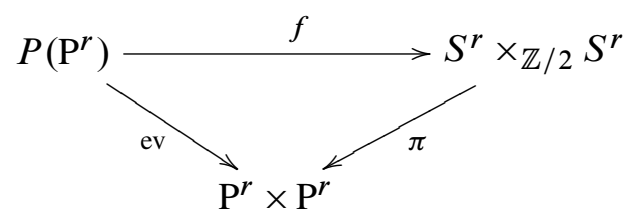

where $\pi$ is the canonical projection, and the $\mathbb{Z} / 2$-action on $S^{r} \times_{\mathbb{Z} / 2} S^{r}$ switches coordinates (and the $\mathbb{Z} / 2$-actions on $P\left(\mathrm{P}^{r}\right)$ and $\mathrm{P}^{r} \times \mathrm{P}^{r}$ are the obvious extensions of the respective $\mathbb{Z} / 2$-actions on $P_{1}\left(\mathrm{P}^{r}\right)$ and $\mathrm{P}^{r} \times \mathrm{P}^{r}-\Delta_{\mathrm{P}^{r}}$ described just before Definition 1.2). In particular, by restricting to $\mathrm{P}^{r} \times \mathrm{P}^{r}-\Delta_{\mathrm{P}}$ and then passing to $\mathbb{Z} / 2$-orbit spaces, (3) yields corresponding triangles:
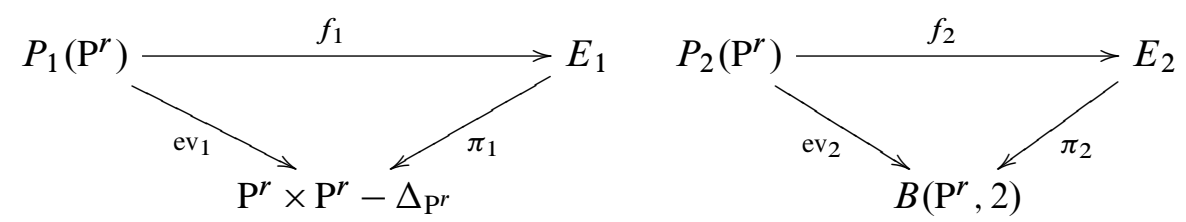

Proposition 2.1 For $i \in\{1,2\}$, genus $\left(\mathrm{ev}_{i}\right)=\operatorname{genus}\left(\pi_{i}\right)$.

Proof It suffices to construct a fiber-preserving $\mathbb{Z} / 2$-equivariant map $g_{1}: E_{1} \rightarrow$ $P_{1}\left(\mathrm{P}^{r}\right)$ running backwards in the left triangle of (4). To this end, we use a straightforward adaptation of the idea in the first part of the proof of [14, Proposition 17]. An 
explicit model for $E_{1}$ is the quotient of $S^{r} \times S^{r}-\tilde{\Delta}$ by the relation $(x, y) \sim(-x,-y)$, where $\widetilde{\Delta} \subset S^{r} \times S^{r}$ is given by $\widetilde{\Delta}=\left\{(x, y) \in S^{r} \times S^{r} \mid x \neq \pm y\right\}$. In these terms, the $\mathbb{Z} / 2$-action on $E_{1}$ interchanges coordinates. Then, the required map $g_{1}$ takes the class of a pair $\left(x_{1}, x_{2}\right)$ into the curve $[0,1] \rightarrow S^{r} \rightarrow \mathrm{P}^{r}$, where the second map is the canonical projection, and the first map is given by

$$
t \mapsto v\left(t x_{2}+(1-t) x_{1}\right) \text {. }
$$

Here $v: \mathbb{R}^{r+1}-\{0\} \rightarrow S^{r}$ is the normalization map.

The main trick in [4] is adapted for the proof of the following result.

Proposition 2.2 If $\rho: \mathrm{P}^{r} \times \mathrm{P}^{r}-\Delta_{\mathrm{P}} r \rightarrow B\left(\mathrm{P}^{r}, 2\right)$ stands for the canonical projection associated to the involution $\left(\mathrm{P}^{r} \times \mathrm{P}^{r}-\Delta_{\mathrm{P}}, \mathbb{Z} / 2\right)$ in Theorem 1.4, then genus $(\rho)=$ $\operatorname{genus}\left(\pi_{2}\right)$.

Proof As indicated in the proof of Proposition 2.1, $E_{2}$ is the quotient of $S^{r} \times S^{r}-\widetilde{\Delta}$ by the relations

$$
(-x,-y) \sim(x, y) \sim(y, x) .
$$

Likewise, the space $\mathrm{P}^{r} \times \mathrm{P}^{r}-\Delta_{\mathrm{P}} r$ is the quotient of $S^{r} \times S^{r}-\tilde{\Delta}$ by the relations

$$
(-x, y) \sim(x, y) \sim(x,-y) .
$$

Moreover, the map

$$
S^{r} \times S^{r}-\tilde{\Delta} \stackrel{\Psi}{\longrightarrow} S^{r} \times S^{r}-\widetilde{\Delta}, \quad \Psi(x, y)=(v(x+y), v(x-y)),
$$

where $v$ is the normalization map at the end of the proof of Proposition 2.1, sends relations (6) into relations (7) and vice versa. Moreover, the resulting maps $\Psi^{\prime}: E_{2} \rightarrow$ $\mathrm{P}^{r} \times \mathrm{P}^{r}-\Delta_{\mathrm{P}} r$ and $\Psi^{\prime \prime}: \mathrm{P}^{r} \times \mathrm{P}^{r}-\Delta_{\mathrm{P}} \rightarrow E_{2}$ are easily seen to be equivariant with respect to the $\mathbb{Z} / 2$-action on $E_{2}$ coming from $\pi_{2}$ in the right triangle of (4) and on $\mathrm{P}^{r} \times \mathrm{P}^{r}-\Delta \mathrm{P}^{r}$ coming from interchanging coordinates.

The result is now a direct consequence of (2).

Proof of Theorem 1.4 Use, in this order, Definition 1.2, Proposition 2.1, Proposition 2.2 and (2). 


\section{Haefliger's metastable range}

Most cases in Theorem 1.3 will follow directly from Theorem 1.4 and the following characterization of smooth embeddings (proved in [17, Théorème $\left.1^{\prime}\right]$ ).

Theorem 3.1 (Haefliger) Let $2 m \geq 3(n+1)$. For a smooth compact $n$-dimensional manifold $M$, there is a surjective map from the set of isotopy classes of smooth embeddings $M \subset \mathbb{R}^{m}$ onto the set of $\mathbb{Z} / 2$-equivariant homotopy classes of maps $M^{*} \rightarrow S^{m-1}$. Here $\mathbb{Z} / 2$ acts antipodally on $S^{m-1}$ and by interchanging coordinates on $M^{*}=M \times M-\Delta_{M}$.

Of course, all we need from Theorem 3.1 is the fact that, under the stated hypothesis (the so-called metastable range), the existence of a smooth embedding $M \subset \mathbb{R}^{m}$ is equivalent to the existence of a $\mathbb{Z} / 2$-equivariant map $M^{*} \rightarrow S^{m-1}$. Although not relevant for our immediate purposes, it is worth remarking that the surjective map in Theorem 3.1 is explicit (see (10)) and that it is in fact bijective when $2 m>3(n+1)$.

Proof of Theorem 1.3 An immediate consequence of Proposition 3.2 below is that the cases with $r \geq 8$ in Theorem 1.3 lie within the metastable range hypothesis in Theorem 3.1. Therefore, in those cases, Theorem 1.3 follows from Theorems 1.4 and 3.1. The few cases in Theorem 1.3 outside Haefliger's metastable range $(r \in\{1,2,4\})$ will be handled at the end of this section. (It would be interesting to know whether any one of the remaining cases $r \in\{3,5,6,7,10,11,12,14,15\}$ gives an actual exception to Theorem 1.3.)

Recall that $\mathrm{P}^{r} \times \mathrm{P}^{r} \rightarrow \mathrm{P}^{s}$ is said to be an axial map if it is homotopically nontrivial over each axis. From [1, Lemma 2.1] we know that, when $r>15$, an axial map $\mathrm{P}^{r} \times \mathrm{P}^{r} \rightarrow \mathrm{P}^{s}$ can exist only for $2 s>3 r$ (in view of Theorem 4.3 below, such an axial map that in addition was symmetric would yield an embedding within Haefliger's metastable range). We will need to consider the following slight improvement.

Proposition 3.2 For $r \in\{8,9,13\}$ or $r>15$, an axial map $\mathrm{P}^{r} \times \mathrm{P}^{r} \rightarrow \mathrm{P}^{s}$ can exist only when $2 s \geq 3(r+1)$.

Proof The main result in [1] (see Theorem 4.1 below) implies that the axial map hypothesis can be replaced by an immersion $\mathrm{P}^{r} \rightarrow \mathbb{R}^{s}$, and we need to prove that, for $r$ as stated, the smallest such $s$ satisfies $2 s>3 r+2$. Cases with $r \in\{8,9,13\}$ follow from inspection of [6]. For $r>15$ we revisit the argument in the proof of [1, Lemma 2.1]. Pick $\rho \geq 4$ with $2^{\rho} \leq r<2^{\rho+1}$. Each of the cases 
- $r \leq 2^{\rho}+3$

- $r=2^{\rho+1}-1$

- $2^{\rho}+2^{\rho-1}+2 \leq r \leq 2^{\rho+1}-3$

can be dealt with by the corresponding nonimmersion result stated in [1].

Assume $2^{\rho}+4 \leq r \leq 2^{\rho}+2^{\rho-1}+1$ and choose $\sigma \in\{1,2, \ldots, \rho-2\}$ with $2^{\rho}+2^{\sigma}+2 \leq$ $r \leq 2^{\rho}+2^{\sigma+1}+1$. From [7], $\mathrm{P}^{2^{\rho}+2^{\sigma}+2}$ does not immerse in Euclidean space of dimension $2^{\rho+1}+2^{\sigma+1}-4$. Therefore, in the optimal immersion $\mathrm{P}^{r} \rightarrow \rightarrow \mathbb{R}^{s}$, we must have $s \geq 2^{\rho+1}+2^{\sigma+1}-3$, and this easily yields the required inequality $2 s>3 r+2$ when $\sigma \geq 3$ or $\rho \geq 5$. For the smaller cases with $\rho=4$ and $1 \leq \sigma \leq 2$, the required $2 s>3 r+2$ follows, as above, from direct inspection of [6].

It remains to consider the case $r=2^{\rho+1}-2$. As the case $\rho=4$ follows again from inspection of [6], we assume further $\rho \geq 5$. Let $m=2^{\rho-1}+2^{\rho-2}+2^{\rho-3}$. From [7] we know that $P^{2(m+\alpha(m)-1)}$ does not immerse $\mathbb{R}^{4 m-2 \alpha(m)}$, where $\alpha(m)$ is the number of ones appearing in the dyadic expansion of $m$. Therefore, in the optimal immersion $\mathrm{P}^{r} \rightarrow \mathbb{R}^{s}$, we must have $s \geq 4 m-2 \alpha(m)+1=2^{\rho+1}+2^{\rho}+2^{\rho-1}-5$, from which one easily deduces the required inequality $2 s>3 r+2$.

We close this section by describing what we know about the numerical value of $\mathrm{TC}^{S}\left(\mathrm{P}^{r}\right)$ for the small values of $r$ not covered by the hypothesis of Proposition 3.2, ie, when Haefliger's metastable range hypothesis in Theorem 1.3 might fail to hold.

The starting point is

$$
\mathrm{TC}\left(\mathrm{P}^{r}\right) \leq \mathrm{TC}^{S}\left(\mathrm{P}^{r}\right) \leq E\left(\mathrm{P}^{r}\right)+1 .
$$

The first inequality has been proved (for any space and not only for projective spaces) in [12, Corollary 9], whereas the second inequality holds without restriction on $r$ in view of Theorem 1.4 and since the construction of the map in Theorem 3.1 makes no use of Haefliger's range (see (10) below). In our current range $r \leq 15$, the precise numeric value of the lower bound for $\mathrm{TC}^{S}\left(\mathrm{P}^{r}\right)$ coming from the first inequality in (9) is determined by $[6 ; 14]$. As for the upper bound, the term $E\left(\mathrm{P}^{r}\right)$ on the right hand side of (9) can even be replaced by the potentially smaller $E_{\mathrm{TOP}}\left(\mathrm{P}^{r}\right)$, the dimension of the smallest Euclidean space where $\mathrm{P}^{r}$ admits a topological embedding. Indeed, such an embedding $g: \mathrm{P}^{r} \hookrightarrow \mathbb{R}^{d}$ determines a $\mathbb{Z} / 2$-equivariant map $\tilde{g}: \mathrm{P}^{r} \times \mathrm{P}^{r}-\Delta_{\mathrm{P}} \rightarrow S^{d-1}$ by the usual formula

$$
\widetilde{g}(a, b)=\frac{g(a)-g(b)}{|g(a)-g(b)|} .
$$


Now, the low dimensional cases under consideration either have $r \leq 7$ or $r=10$, $11,12,14,15$. For $r=7$ and $r=15$ the use of $E_{\mathrm{TOP}}\left(\mathrm{P}^{r}\right)$ gives more accurate information than that available for $E\left(\mathrm{P}^{r}\right)$. Indeed, the PL embeddings $\mathrm{P}^{7} \hookrightarrow \mathbb{R}^{10}$ and $\mathrm{P}^{15} \hookrightarrow \mathbb{R}^{23}$ constructed in [23] improve by 2 and 1 units, respectively, the upper bound in (9) obtained from the best smooth embedding results currently known [6]. Also worth noticing is the fact that Rees' upper bound

$$
\operatorname{level}\left(\mathrm{P}^{6} \times \mathrm{P}^{6}-\Delta_{\mathrm{P}^{6}}, \mathbb{Z} / 2\right) \leq 9,
$$

obtained in [22, Corollary 11], improves by 2 units the upper bound in (9). On the other hand, it is elementary to check that, in all cases with $r \leq 7$, [12, Theorem 17] improves by one unit the lower bound in (9).

Table 1 summarizes the resulting improved bounds $\ell(r) \leq \mathrm{TC}^{S}\left(\mathrm{P}^{r}\right) \leq u(r)$. Note that the case $r=4$ does lie within Haefliger's metastable range and together with the cases $r=1,2$ gives the three missing instances in the proof of Theorem 1.3.

\begin{tabular}{|c|c|c|c|c|c|c|c|c|c|c|c|c|}
\hline$r$ & 1 & 2 & 3 & 4 & 5 & 6 & 7 & 10 & 11 & 12 & 14 & 15 \\
\hline$u(r)$ & 3 & 5 & 6 & 9 & 10 & 10 & 11 & 18 & 19 & 22 & 24 & 24 \\
\hline$\ell(r)$ & 3 & 5 & 5 & 9 & 9 & 9 & 9 & 17 & 17 & 19 & 23 & 23 \\
\hline
\end{tabular}

Table 1: Upper and lower bounds for $\mathrm{TC}^{S}\left(\mathrm{P}^{r}\right)$ for low values of $r$

Examples 3.3 Let $\delta=(0,1,2), i \geq(1,3,4)$ and $r=2^{i}+\delta$. According to [6] and Theorems 1.3 and 4.2 (below), we have

$$
\mathrm{TC}^{S}\left(\mathrm{P}^{r}\right)-\mathrm{TC}\left(\mathrm{P}^{r}\right)=(1,2,1) .
$$

This situation contrasts with the fact, proved in Section 6, that the first inequality in (9) becomes an equality for all complex projective spaces. Actually, in view of the main result in [20], there is even a (weird) possibility that the left hand side of (11) might turn out to be an unbounded function of $r$ (compare to [12, Example 28]). Other situations with a behavior resembling that in (11) are given by spheres: according to [9; 12], $\mathrm{TC}^{S}\left(S^{r}\right)-\mathrm{TC}\left(S^{r}\right)=0$ for $n$ even, whereas $\mathrm{TC}^{S}\left(S^{r}\right)-\mathrm{TC}\left(S^{r}\right)=1$ for $n$ odd.

\section{Relation to axial maps}

In Sections 1 and 2 we made it clear that, in characterizing the embedding dimension for real projective spaces, one might prefer to avoid the use of symmetric axial maps. However, in this section we analyze the way Theorem 1.3 is related to such maps. 
Our justification for including this section is three-fold. First, it shows how our proof of Theorem 1.3 arose (compare the map $\Psi$ in (13) below with that in (8)). Second, it illustrates the use (and, as observed in the next section, the limitations) of the constructions in Section 4.2 when applied to the case of even-torsion lens spaces. And third, this material will allow us to make explicit comparisons with the maps arising in the next section (eg, (17), (18) and (19) as generalized forms of (12)) towards a characterization of the symmetric topological complexity of lens spaces (Theorem 5.3 and Proposition 5.5).

The section has been divided into three short subsections. We start with a brief review of the axial map interpretation for the immersion problem of real projective spaces (Section 4.1) and the corresponding (partial) interpretation known before this paper for embeddings (Section 4.2). The main goal then is to compare our methods in Section 2 to those in [4] (Section 4.2) and [14] (Section 4.3).

There are no new results in this section; instead, it has a retrospective flavor, written much in the way the ideas in this paper originally arose. The reader interested in our analysis and results on the symmetric topological complexity of lens spaces and complex projective spaces can safely skip this section and proceed directly to the final Sections 5 and 6, respectively.

\subsection{Axial maps, immersions and topological complexity}

Activities were launched with Hopf's early work [19] constructing, for $n>r$, a Euclidean $n$-dimensional embedding for $\mathrm{P}^{r}$ from a given symmetric nonsingular bilinear map $\alpha: \mathbb{R}^{r+1} \times \mathbb{R}^{r+1} \rightarrow \mathbb{R}^{n+1}$. By restricting to unit vectors (and normalizing), this yields a symmetric $\mathbb{Z} / 2$-biequivariant map $\widetilde{\alpha}: S^{r} \times S^{r} \rightarrow S^{n}$, ie, one satisfying conditions (12) below. Note that $\tilde{\alpha}$ covers an axial map $\hat{\alpha}: \mathrm{P}^{r} \times \mathrm{P}^{r} \rightarrow \mathrm{P}^{n}$ that, in addition, is symmetric in the sense that the relation $\widehat{\alpha}(a, b)=\widehat{\alpha}(b, a)$ holds for $a, b \in \mathrm{P}^{r}$. Using Hirsch's characterization of smooth Euclidean immersions in terms of the geometric dimension of the normal bundle, the relevance of (not necessarily symmetric) axial maps was settled in [1] by showing:

Theorem 4.1 For $n>r$, the existence of an axial map $\mathrm{P}^{r} \times \mathrm{P}^{r} \rightarrow \mathrm{P}^{n}$ is equivalent to the existence of a smooth immersion $\mathrm{P}^{r} \rightarrow \mathbb{R}^{n}$.

The hypothesis $n>r$ is needed only for $r=1,3,7$. In those cases $\mathrm{P}^{r}$ is parallelizable and has an optimal Euclidean immersion in codimension 1; however the complex, quaternion and octonion multiplications yield axial maps with $n=r$.

But the connection with robotics was established after 30 years with M Farber's work (initiated in $[8 ; 9]$ ) on the motion planning problem. The main result in [14] is: 
Theorem 4.2 For $r \neq 1,3,7, \mathrm{TC}\left(\mathrm{P}^{r}\right)$ is the smallest integer $n$ such that there is an axial map $\mathrm{P}^{r} \times \mathrm{P}^{r} \rightarrow \mathrm{P}^{n-1}$. Consequently, $\mathrm{TC}\left(\mathrm{P}^{r}\right)-1$ is the smallest dimension of Euclidean spaces where $\mathrm{P}^{r}$ can be smoothly immersed. This assertion holds for the three exceptional values of $r$ provided $\mathrm{TC}\left(\mathrm{P}^{r}\right)-1$ is replaced by $\mathrm{TC}\left(\mathrm{P}^{r}\right)$.

\subsection{Symmetric axial maps and embeddings}

As shown in [4], the embedding problem for real projective spaces can be closely modeled by keeping Hopf's original symmetry condition for axial maps. We give below a quick review of some of the main ideas in [4].

Start by observing that a symmetric axial map $\hat{\alpha}: \mathrm{P}^{r} \times \mathrm{P}^{r} \rightarrow \mathrm{P}^{s}$ is covered by a map $\tilde{\alpha}: S^{r} \times S^{r} \rightarrow S^{s}$ satisfying

$$
-\tilde{\alpha}(x, y)=\tilde{\alpha}(-x, y)=\tilde{\alpha}(x,-y) \text { and } \tilde{\alpha}(x, y)=\tilde{\alpha}(y, x)
$$

for $x, y \in S^{r}$. Under these conditions it is elementary to check that the composite

$$
V_{r+1,2} \stackrel{\Psi}{\longrightarrow} S^{r} \times S^{r} \stackrel{\tilde{\alpha}}{\longrightarrow} S^{s}, \quad \Psi(x, y)=\left(\frac{x+y}{\sqrt{2}}, \frac{x-y}{\sqrt{2}}\right)
$$

is a $D_{4}$-equivariant map. Here $D_{4}$ is the dihedral group written as the wreath product $(\mathbb{Z} / 2 \times \mathbb{Z} / 2) \rtimes \mathbb{Z} / 2$ where $\mathbb{Z} / 2$ acts on $\mathbb{Z} / 2 \times \mathbb{Z} / 2$ by interchanging factors. This group acts on $S^{s}$ via the canonical projection $(\mathbb{Z} / 2 \times \mathbb{Z} / 2) \rtimes \mathbb{Z} / 2 \rightarrow \mathbb{Z} / 2$, and on $V_{r+1,2}$ (the Stiefel manifold of orthonormal 2-frames in $\mathbb{R}^{r+1}$ ) via the restricted left $D_{4}$-action in $S^{r} \times S^{r}$, where $\mathbb{Z} / 2 \times \mathbb{Z} / 2$ and $\mathbb{Z} / 2$ act on $S^{r} \times S^{r}$ by the product antipodal-action and by switching coordinates, respectively.

On the other hand, with the notation $\widetilde{\Delta}=\left\{(x, y) \in S^{r} \times S^{r} \mid x \neq \pm y\right\}$ in the proof of Proposition 2.1, the map $H:\left(S^{r} \times S^{r}-\widetilde{\Delta}\right) \times[0,1] \rightarrow S^{r} \times S^{r}-\widetilde{\Delta}$ defined by $H\left(u_{1}, u_{2}, t\right)=\left(\tilde{u}_{1}, \tilde{u}_{2}\right)$ where

$$
\begin{aligned}
\tilde{u}_{1} & =\frac{u_{1}+t\left(v_{1}-u_{1}\right)}{\left\|u_{1}+t\left(v_{1}-u_{1}\right)\right\|} & \tilde{u}_{2} & =\frac{u_{2}+t\left(v_{2}-u_{2}\right)}{\left\|u_{2}+t\left(v_{2}-u_{2}\right)\right\|} \\
v_{1} & =w_{1}+w_{2} & v_{2} & =w_{1}-w_{2} \\
w_{1} & =\frac{u_{1}+u_{2}}{\sqrt{1+\left\langle u_{1}, u_{2}\right\rangle}} & w_{2} & =\frac{u_{1}-u_{2}}{\sqrt{1-\left\langle u_{1}, u_{2}\right\rangle}}
\end{aligned}
$$

gives a $D_{4}$-equivariant deformation retraction of $S^{r} \times S^{r}-\tilde{\Delta}$ onto $V_{r+1,2}$. (Figure 1 depicts the case in which the angle between $u_{1}$ and $u_{2}$ is less than 90 degrees; the situation for an angle between 90 and 180 degrees is similar, but lowering the angle to be 90 degrees.) Then, composing the retraction $H(-, 1)$ with $\tilde{\alpha} \circ \Psi$ and passing to $(\mathbb{Z} / 2 \times \mathbb{Z} / 2)$-orbit spaces, we get a $\mathbb{Z} / 2$-equivariant map $\left(\mathrm{P}^{r}\right)^{*} \rightarrow S^{s}$. 


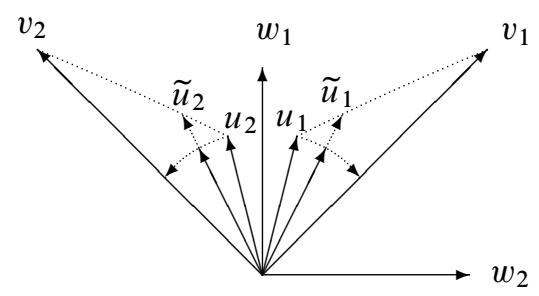

Figure 1: The $D_{4}$-equivariant deformation retraction $H$

In view of Theorem 3.1, the above construction settles the first statement of Theorem 4.3 below. The bulk of the work in [4] uses Haefliger and Hirsch's fundamental work [17; 18] on embeddings and immersions in the stable range to establish the second statement of Theorem 4.3.

Theorem 4.3 The existence of a symmetric axial map $\mathrm{P}^{r} \times \mathrm{P}^{r} \rightarrow \mathrm{P}^{s}$ implies the existence of a smooth embedding $\mathrm{P}^{r} \subset \mathbb{R}^{s+1}$, provided $2 s>3 r$. The existence of a smooth embedding $\mathrm{P}^{r} \subset \mathbb{R}^{s}$ implies the existence of a symmetric axial map $\mathrm{P}^{r} \times \mathrm{P}^{r} \rightarrow \mathrm{P}^{s}$.

The arguments in [4] go a bit further. Using the full power of Theorem 3.1, it is possible to explicitly relate, for instance, isotopy classes of embeddings to symmetric homotopy classes of symmetric axial maps. We will not make use of these more complete results, though.

Theorem 4.3 can be interpreted as follows. Let $a_{S}(r)$ denote the smallest integer $k$ for which there exists a symmetric axial map $\mathrm{P}^{r} \times \mathrm{P}^{r} \rightarrow \mathrm{P}^{k}$. It is immediate from Theorem 4.3 that, at least for $r$ as in Proposition 3.2,

$$
E(r)=a_{S}(r)+\delta \text { with } \delta=\delta(r) \in\{0,1\} .
$$

To the best of our knowledge, the explicit value of $\delta$ (as a function of $r$ ) remains an open question. As explained in Section 1, Theorems 1.3 and 1.4 avoid this $\delta$ indeterminacy by replacing $a_{S}(r)$ with $\mathrm{TC}^{S}\left(\mathrm{P}^{r}\right)$.

\subsection{Symmetric axial maps and symmetric TC}

The goal of this section is to offer a direct comparison between our methods and those used in [14] for the nonsymmetric case. To this end, we start by observing the following obvious consequence of (14) and the second inequality in (9).

Corollary 4.4 For any $r, \mathrm{TC}^{S}\left(\mathrm{P}^{r}\right) \leq a_{S}(r)+2$. 
In view of Theorem 1.3, this can be thought of as extending the $E$ vs. $a_{S}$ relation in (14) within the topological complexity viewpoint. Of course, for $r$ as in Theorem 1.3 , the above inequality is within one unit of being an equality.

Proof Corollary 4.4 can be settled with a straightforward adaptation of the idea in the first part of the proof of [14, Proposition 17]. Namely, let $\tilde{\alpha}: S^{r} \times S^{r} \rightarrow S^{s}$ satisfy (12), with $s=a_{S}(r)$. For $1 \leq i \leq s+1$, let $\alpha_{i}: S^{r} \times S^{r} \rightarrow \mathbb{R}$ be the $i$-th real component of $\tilde{\alpha}$, and set $U_{i}$ to be the open subset of $\mathrm{P}^{r} \times \mathrm{P}^{r}-\Delta_{\mathrm{P}} r$ consisting of pairs $\left(L_{1}, L_{2}\right)$ of lines with $\alpha_{i}\left(\ell_{1}, \ell_{2}\right) \neq 0$, for representatives $\ell_{j} \in L_{j} \cap S^{r}, j=1$, 2. Consider the function $s_{i}: U_{i} \rightarrow P_{1}\left(\mathrm{P}^{r}\right)$ defined as follows: given $\left(L_{1}, L_{2}\right) \in U_{i}$, choose elements $\ell_{j}$ as above with $\alpha_{i}\left(\ell_{1}, \ell_{2}\right)>0$. The two such possibilities $\left(\ell_{1}, \ell_{2}\right)$ and $\left(-\ell_{1},-\ell_{2}\right)$ give the same orientation for the 2-plane $P\left(L_{1}, L_{2}\right)$ generated by $L_{1}$ and $L_{2}$. Under these conditions, $s_{i}\left(L_{1}, L_{2}\right)$ is the path rotating $L_{1}$ to $L_{2}$ in the oriented plane $P\left(L_{1}, L_{2}\right)$.

Evidently $s_{i}$ is a continuous section of the fibration $\mathrm{ev}_{1}$ over $U_{i}$. It is also $\mathbb{Z} / 2-$ equivariant, in view of the last condition in (12). Therefore, it induces a corresponding (continuous) section $\overline{s_{i}}$ of the fibration $\mathrm{ev}_{2}$ over the image of $U_{i}$ under the canonical (open) projection $\mathrm{P}^{r} \times \mathrm{P}^{r}-\Delta \mathrm{P}^{r} \rightarrow B\left(\mathrm{P}^{r}, 2\right)$. But $\mathrm{P}^{r} \times \mathrm{P}^{r}-\Delta_{\mathrm{P}} r$ is covered by the $U_{i}$ 's, so we deduce genus $\left(\mathrm{ev}_{2}\right) \leq s+1$. Adding 1 gives the conclusion in Corollary 4.4.

Next, we elaborate on the main difference between our methods and those in [14]. Let $\xi$ be the Hopf line bundle over $\mathrm{P}^{r}$ and consider the exterior tensor product $\xi \otimes \xi$ over $\mathrm{P}^{r} \times \mathrm{P}^{r}$. Let $I(r)$ denote the smallest integer $k$ such that the iterated $(k+1)-$ fold Whitney multiple of $\xi \otimes \xi$ admits a nowhere vanishing section. Finally, let $a(r)$ denote the smallest integer $k$ for which there is a (perhaps nonsymmetric) axial map $\mathrm{P}^{r} \times \mathrm{P}^{r} \rightarrow \mathrm{P}^{k}$. The main results in [14], Corollary 5 and Proposition 17, give

$$
I(r)+1 \leq T C\left(\mathrm{P}^{r}\right) \leq a(r)+1,
$$

an assertion a bit sharper than its symmetric analogue (Theorem 1.3 and Corollary 4.4). The punch line then comes from the classical fact that, for $n \neq 1,3,7$, both $I(r)$ and $a(r)$ agree with the dimension of the smallest Euclidean space where $\mathrm{P}^{r}$ admits a smooth immersion (in the case of $I(r)$ see, for instance, the proof of [16, Proposition 2.7]). However, there is no sectioning-Whitney-multiples interpretation available for a symmetric version of (15). Instead, as detailed below, the solution comes from an adaptation of the ideas in [4].

Recall the Borel construction $S^{r} \times_{\mathbb{Z} / 2} S^{r}$ introduced in Section 2. The 2-fold Cartesian product of the canonical projection $S^{r} \rightarrow \mathrm{P}^{r}$ factors through $S^{r} \times_{\mathbb{Z} / 2} S^{r}$ yielding the $\mathbb{Z} / 2$-covering space $\pi: S^{r} \times \mathbb{Z} / 2 S^{r} \rightarrow \mathrm{P}^{r} \times \mathrm{P}^{r}$ in (3). It is well known that $\pi$ is the sphere bundle associated to $\xi \otimes \xi \rightarrow \mathrm{P}^{r} \times \mathrm{P}^{r}$ (see for instance [26, Lemma 3.1]). 
The relevance of such an observation comes from [24, Theorem 3 and final remarks in Chapter II], which affirms that the Whitney multiple $k(\xi \otimes \xi)$ admits a global nowhere zero section for $k=\operatorname{genus}(\pi)$, that is, $I(r)+1 \leq \operatorname{genus}(\pi)$. In these terms, the work in [14] for settling the first inequality in (15) comes from observing that the topological complexity of $\mathrm{P}^{r}$ is bounded from below by genus $(\pi)$. (The second inequality in (15) is actually settled in [14] with a sharpening of the argument we gave above for proving Corollary 4.4.) This lower bound is easily settled in [14, Theorem 3] from diagram (3). In fact, as a byproduct of the methods in [14], it is known that

$$
\operatorname{TC}\left(\mathrm{P}^{r}\right)=\operatorname{genus}(\pi) .
$$

But, in order to get up to this key stage in the symmetric situation, we needed to adapt the main trick in [4]. Indeed, as shown in Section 2, Propositions 2.1 and 2.2 provide us with the needed substitute for (16), from which the proof of Theorems 1.3 and 1.4 easily follows.

\section{Lens spaces}

Unlike the case of real projective spaces, the symmetric topological complexity of a lens space $L^{2 n+1}(m)$ - the orbit space of the standard $\mathbb{Z} / m$-action on $S^{2 n+1}$-is in general not related to its embedding dimension nor, for that matter, to the level of the switching involution on $L^{2 n+1}(m) \times L^{2 n+1}(m)-\Delta_{L^{2 n+1}(m)}$. And here is an extreme example: while the high-torsion lens spaces $L^{2 n+1}(m)$ in Section 5.3 have symmetric topological complexity equal to $4 n+\epsilon$, with $\epsilon \in\{2,3\}$ (see (22)), the level of the corresponding switching involution is at most $2 n+3$, for all odd $m$ (see Rees [22]).

In retrospect, the problem arises (in the 2-local situation) from the fact that the (nonsymmetric) topological complexity of $L^{2 n+1}\left(2^{e}\right)$ actually differs from the immersion dimension of this manifold, and the difference gets larger as $e$ increases, until it attains a certain stable value (see the remark following Theorem 5.7).

Following the nonsymmetric lead, in this section we (a) indicate how one can characterize the symmetric topological complexity of an even-torsion lens space (Theorem 5.3 and Proposition 5.5) and (b) point out concrete differences with respect to a similar characterization for its embedding dimension. To better appreciate the picture, it will be convenient to start by making a summary of, and comparing to, the known situation in the nonsymmetric case. 


\section{$5.1 e$-axial maps, immersions and embeddings of $L^{2 n+1}\left(2^{e}\right)$}

The well known relation (Theorem 4.1) between Euclidean immersions of real projective spaces and (not necessarily symmetric) axial maps has been generalized in [2] for $2^{e}$-torsion lens spaces to prove that, with the possible exceptions of $n=2,3,5$, the existence of an immersion $L^{2 n+1}\left(2^{e}\right) \rightarrow \rightarrow \mathbb{R}^{m}$ is equivalent to the existence of an $e-$ axial map $\mathrm{P}^{2 n+1} \times \mathbb{Z} / 2^{e-1} \mathrm{P}^{2 n+1} \rightarrow \mathrm{P}^{m}$, that is, a map that yields a standard axial map when precomposed with the canonical projection $\mathrm{P}^{2 n+1} \times \mathrm{P}^{2 n+1} \rightarrow \mathrm{P}^{2 n+1} \times \mathbb{Z} / 2^{e-1}$ $\mathrm{P}^{2 n+1}$. Here the notation $\mathrm{P}^{2 n+1} \times_{\mathbb{Z} / 2^{e-1}} \mathrm{P}^{2 n+1}$ refers to the usual Borel construction with respect to the standard free $\mathbb{Z} / 2^{e-1}$-action on $\mathrm{P}^{2 n+1}$ with orbit space $L^{2 n+1}\left(2^{e}\right)$.

At the level of covering spaces, the $e$-axial map condition translates into having a map $\tilde{\alpha}: S^{2 n+1} \times S^{2 n+1} \rightarrow S^{m}$ satisfying the relations

$$
\widetilde{\alpha}(\omega x, y)=\tilde{\alpha}(x, \omega y) \text { and } \tilde{\alpha}(-x, y)=-\widetilde{\alpha}(x, y)
$$

for $x, y \in S^{2 n+1}$ and $\omega \in \mathbb{Z} / 2^{e} \subset S^{1}$-these correspond to the first group of conditions in (12). Our first objective is to indicate how the slight variation in (18) below of the obvious symmetrization of these conditions describes the Euclidean embedding dimension for (arbitrary-torsion) lens spaces.

For an integer $m \geq 2$, the product action of $\mathbb{Z} / m \times \mathbb{Z} / m$ on the Cartesian product $S^{2 n+1} \times S^{2 n+1}$ extends to a left action of the wreath product $G_{m}=(\mathbb{Z} / m \times \mathbb{Z} / m) \rtimes$ $\mathbb{Z} / 2$, where $\mathbb{Z} / 2$ acts on $S^{2 n+1} \times S^{2 n+1}$ by interchanging axes. This action is stable on the orbit configuration space $F_{\mathbb{Z} / m}\left(S^{2 n+1}, 2\right)$ consisting of pairs in $S^{2 n+1} \times S^{2 n+1}$ generating different $\mathbb{Z} / m$-orbits (this is the obvious generalization of the space $S^{r} \times S^{r}-\widetilde{\Delta}$ found in Section 4.2 as well as in the proofs of Propositions 2.1 and 2.2). The quotient $F_{n, m}=F_{\mathbb{Z} / m}\left(S^{2 n+1}, 2\right) /(\mathbb{Z} / m \times \mathbb{Z} / m)$ has an involution induced by the action of $G_{m}$ on the orbit configuration space, and this gives a $\mathbb{Z} / 2$-equivariant model for $L^{2 n+1}(m) \times L^{2 n+1}(m)-\Delta_{L^{2 n+1}(m)}$, where $\mathbb{Z} / 2$ acts by switching coordinates. In these terms, Theorem 3.1 translates into:

Lemma 5.1 Assume $k \geq 3(n+1)$. $L^{2 n+1}(m)$ can be smoothly embedded in $\mathbb{R}^{k}$ if and only if there is a $\mathbb{Z} / 2$-equivariant map $F_{n, m} \rightarrow S^{k-1}$.

Of course, having a $\mathbb{Z} / 2$-equivariant map as above is equivalent to having a $G_{m}-$ equivariant map $\widetilde{\alpha}: F_{\mathbb{Z} / m}\left(S^{2 n+1}, 2\right) \rightarrow S^{k-1}$, where $G_{m}$ acts on $S^{k-1}$ via the canonical projection $(\mathbb{Z} / m \times \mathbb{Z} / m) \rtimes \mathbb{Z} / 2 \rightarrow \mathbb{Z} / 2$. Explicitly, $\tilde{\alpha}$ must satisfy

$$
\widetilde{\alpha}(\omega x, y)=\tilde{\alpha}(x, y)=\widetilde{\alpha}(x, \omega y) \text { and } \tilde{\alpha}(x, y)=-\tilde{\alpha}(y, x)
$$

for $x, y \in S^{2 n+1}$ and $\omega \in \mathbb{Z} / m \subset S^{1}$. As shown in Section 4.2, in the case $m=2$, the key connection between (18) and the symmetrized version of (17) is given by the 
ideas in [4], which teach us how to take care of the deleted "equivariant diagonal" in $F_{\mathbb{Z} / 2}\left(S^{2 n+1}, 2\right)$. Unfortunately, we have not succeeded in obtaining such a connection for larger values of $m$. The major problem seems to be given by the apparent lack ${ }^{1}$ of a suitable equivariant deformation retraction of $L^{2 n+1}(m) \times L^{2 n+1}(m)-\Delta_{L^{2 n+1}(m)}$ that plays the role of $V_{2 n+2,2}$ in the $m=2$ arguments of [4] described in Section 4.2. It is worth remarking that, in the symmetric $m=2$ situation of Section 2, we do make an indirect use - through the map $\Psi$ in (8) —of this equivariant deformation retraction. This problem will reappear, in a slightly different form, in regard to a potential characterization for the symmetric topological complexity of $m$-torsion lens spaces in terms of the $\mathbb{Z} / m$-biequivariant maps of the next subsection (see the remark following Corollary 5.4).

\subsection{Symmetric biequivariant maps and $\mathrm{TC}^{S}$ of lens spaces}

As shown in [16], when $m$ is even the (nonsymmetric) topological complexity of $L^{2 n+1}(m)$ turns out to be (perhaps one more than) the smallest odd integer $k$ for which there is a $\mathbb{Z} / m$-biequivariant map $\tilde{\alpha}: S^{2 n+1} \times S^{2 n+1} \rightarrow S^{k}$, that is, a map satisfying the (stronger than (17)) requirements

$$
\tilde{\alpha}(\omega x, y)=\tilde{\alpha}(x, \omega y)=\omega \widetilde{\alpha}(x, y),
$$

for $x, y \in S^{2 n+1}$ and $\omega \in \mathbb{Z} / m \subset S^{1}$. Alternatively, if $c: S^{2 n+1} \rightarrow S^{2 n+1}$ stands for complex conjugation in every complex coordinate, then by precomposing with $1 \times c$, a $\mathbb{Z} / m$-biequivariant map as above can equivalently be defined through the requirements

$$
\widetilde{\alpha}(\omega x, y)=\omega \widetilde{\alpha}(x, y)=\widetilde{\alpha}\left(x, \omega^{-1} y\right) .
$$

In analogy to the $a_{S}$ notation introduced at the end of Section 4.2 to measure the existence of symmetric axial maps, the following definition (which, up to composition with $1 \times c$, corresponds to the symmetrized version of the number $s_{n, m}$ defined in [16]) is intended to measure the existence of symmetric $\mathbb{Z} / m$-biequivariant maps.

Definition 5.2 For integers $n$ and $m$, denote by $b_{n, m}^{S}$ the smallest integer $k$ such that there is a map $\tilde{\alpha}: S^{2 n+1} \times S^{2 n+1} \rightarrow S^{2 k-1}$ satisfying (19) and

$$
\widetilde{\alpha}(x, y)=\widetilde{\alpha}(y, x)
$$

for $x, y \in S^{2 n+1}$ and $\omega \in \mathbb{Z} / m \subset S^{1}$.

\footnotetext{
${ }^{1}$ Using the gradient flow navigation technique in [11, Section 4.4], Armindo Costa's current PhD work at Durham University offers a nice explanation of the way this problem arises.
} 
The next result gives our characterization for (half the value of) the symmetric topological complexity of even-torsion lens spaces. The proof will be postponed to the end of this section.

Theorem 5.3 For even $m$, the integral part of $\frac{1}{2} \mathrm{TC}^{S}\left(L^{2 n+1}(m)\right)$ agrees with the smallest integer $k$ such that there is a map $\tilde{\alpha}: F_{\mathbb{Z} / m}\left(S^{2 n+1}, 2\right) \rightarrow S^{2 k-1}$ satisfying (19) and (20) for $x, y \in S^{2 n+1}$ and $\omega \in \mathbb{Z} / m \subset S^{1}$.

Most of the work in [12] goes in the direction of giving strong lower bounds for $\mathrm{TC}^{S}$. However, there seems to be a relative lack of suitable upper bounds; the only ones ${ }^{2}$ we are aware of are derived, some way or other, from Schwarz's general estimate for the genus of a fibration $F \rightarrow E \rightarrow B$ in terms of the dimension of $B$ and the connectivity of $F$ [24, Theorems 5 and $\left.5^{\prime}\right]$. For instance, in [12, Proposition 10] the upper bound

$$
\mathrm{TC}^{S}(M) \leq 2 d+1
$$

is derived for any $d$-dimensional closed smooth manifold $M$. In the case $M=$ $L^{2 n+1}(m)$, Corollary 5.4 below (which is an immediate consequence of Theorem 5.3) offers an alternative to (21) that takes not only dimension into account, but also torsion. Theorem 5.8 below gives a typical example (in the nonsymmetric setting, though) of the potential use of this kind of result.

Corollary 5.4 For even $m$, the integral part of $\frac{1}{2} \mathrm{TC}^{S}\left(L^{2 n+1}(m)\right)$ is no greater than $b_{n, m}^{S}$.

Remark In the direction of exploring a possible symmetric analogue of the main result in [16], it would be useful to make precise how much the above upper bound differs from being an equality. The main obstruction to such a goal seems to be the apparent lack of an analogue for lens spaces of the map $\Psi$ in (8) and (13).

We close this section with the proof of Theorem 5.3. As will quickly become clear, the details are formally the same as in the $m=2$ case. The $m$-analogue of (3), first considered in [14, Theorem 3], reads:

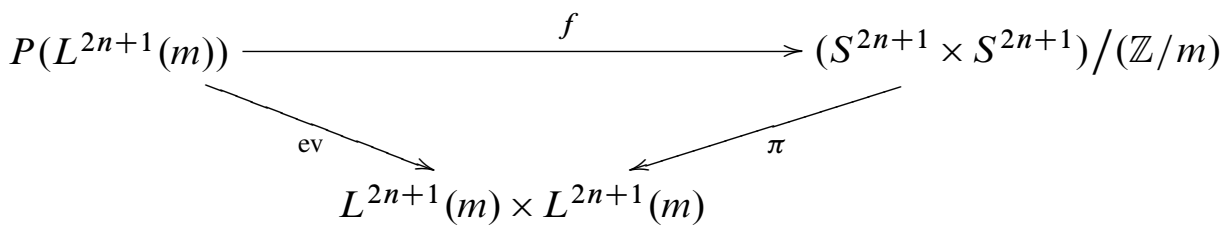

\footnotetext{
${ }^{2}$ The relativized notion of topological complexity [11, Sections 4.3 and 4.4] seems to lead to new such upper bounds.
} 
The orbit space in the upper right corner is taken with respect to the diagonal $\mathbb{Z} / m-$ action. The map $f$, whose definition is the obvious generalization of that in the case $m=2$, is $\mathbb{Z} / 2$-equivariant. In these conditions, the analogue of (4) and the proof of Proposition 2.1 generalize in a straightforward way to produce the following characterization of $\mathrm{TC}^{S}\left(L^{2 n+1}(m)\right)$.

Proposition 5.5 For even $m$,

$$
\operatorname{TC}^{S}\left(L^{2 n+1}(m)\right)-1=\operatorname{genus}\left(\pi_{2, m}: E_{2, m} \longrightarrow B\left(L^{2 n+1}(m), 2\right)\right) .
$$

Here $E_{2, m}$ is the quotient of $F_{\mathbb{Z} / m}\left(S^{2 n+1}, 2\right)$ by the two relations $(x, y) \sim(\omega x, \omega y)$ and $(x, y) \sim(y, x)$. Moreover, $\pi_{2, m}$ is a $\mathbb{Z} / m$-cover with $\mathbb{Z} / m$ acting on $E_{2, m}$ as $\omega \cdot[x, y]=[\omega x, y]$, for $x, y \in S^{2 n+1}$ and $\omega \in \mathbb{Z} / m \subset S^{1}$, where $[x, y]$ stands for the class of the pair $(x, y)$.

Remark The requirement that $m$ be even is used in the construction of the map $g_{1}$ in the proof of Proposition 2.1. The coordinates of a pair $\left(x_{1}, x_{2}\right) \in F_{\mathbb{Z} / m}\left(S^{2 n+1}, 2\right)$ cannot be antipodal when $m$ is even, so that (5) is well defined.

Theorem 5.3 is now a direct consequence of Proposition 5.5 and the following $m-$ analogue of (2) (proved in full generality in [24, Corollary 1, page 97]).

Lemma 5.6 The canonical $\mathbb{Z} / m$-cover $S^{2 n-1} \rightarrow L^{2 n-1}(m)$ classifies $\mathbb{Z} / m$-covers of genus at most $2 n$.

\section{3 (Nonsymmetric) TC of high-torsion lens spaces}

For an integer $m \geq 2$ we say that a lens space $L^{2 n+1}(m)$ is of high torsion when $m$ does not divide the binomial coefficient $\left(\begin{array}{c}2 n \\ n\end{array}\right)$. A lens space that is not of high torsion will be said to be of low torsion. The (nonsymmetric) topological complexity of a high-torsion lens space has recently been settled in [13].

Theorem 5.7 (Farber-Grant) For a high-torsion lens space,

$$
\operatorname{TC}\left(L^{2 n+1}(m)\right)=4 n+2 .
$$

Remark This result is the analogue of the following (2-local) situation. For a fixed $n$, the immersion dimension of $L^{2 n+1}\left(2^{e}\right)$ is a bounded nondecreasing function of $e$ which, therefore, becomes stable for large $e$. As explained in [15] and [16, Section 6], the stable value of the immersion dimension is expected to be attained roughly when $2^{e}$ does not divide $\left(\begin{array}{c}2 n \\ n\end{array}\right)$-with an expected value close to the immersion dimension of the complex projective $n$-dimensional space. A very concrete situation, which compares TC to the immersion dimension of lens spaces, is illustrated in Example 5.10 below. 
The converse implication in the statement of Theorem 5.7 is true when $m$ is even. In fact, we extend Farber-Grant's result to the first case outside the high-torsion range by combining the techniques in [13] with the $\mathbb{Z} / m$-biequivariant map characterization of $\operatorname{TC}\left(L^{2 n+1}(m)\right)$ discussed at the beginning of Section 5.2. The result arose from an e-mail exchange, dating back to mid 2007, between the first author and Professor Farber concerning the results in [13].

Theorem 5.8 Let $m$ be even. If $L^{2 n+1}(m)$ is of low torsion, then $\operatorname{TC}\left(L^{2 n+1}(m)\right) \leq$ $4 n$, with equality when $m$ does not divide $\left(\begin{array}{c}2 n-1 \\ n\end{array}\right)$.

Proof Proposition 2.2 and Theorem 2.9 in [16] yield $\mathrm{TC}\left(L^{2 n+1}(m)\right) \leq 4 n$. The rest comes from [13, Theorem 11] (with $k=n$ and $\ell=n-1$ ).

Since $\left(\begin{array}{c}2 n \\ n\end{array}\right)=2\left(\begin{array}{c}2 n-1 \\ n\end{array}\right)$, the final part in the statement of Theorem 5.8 refers to a 2-local property of $m$, namely, that the highest exponent of 2 in $m$ agrees with that in $\left(\begin{array}{c}2 n \\ n\end{array}\right)$.

Example 5.9 It is well known that the highest power of 2 dividing $\left(\begin{array}{c}2 n \\ n\end{array}\right)$ is $\alpha(n)$, the number of ones in the dyadic expansion of $n$. In particular, $\operatorname{TC}\left(L^{2 n+1}\left(2^{e}\right)\right)=$ $4 n+2$ for $e>\alpha(n)$. Theorem 5.8 now gives $\operatorname{TC}\left(L^{2 n+1}\left(2^{\alpha(n)}\right)\right)=4 n$. Since $\mathrm{TC}\left(L^{2 n+1}(2)\right)$ is (one more than) the immersion dimension of the real projective space $L^{2 n+1}(2)$ (Theorem 4.2), it is highly desirable to get as much information as possible on the value of $\operatorname{TC}\left(L^{2 n+1}\left(2^{e}\right)\right)$ as $e$ goes from $\alpha(n)-1$ down to 1 .

Example 5.10 Table 2 summarizes the topological complexity and immersion dimension for $L^{2 n+1}\left(2^{e}\right)$ and $\mathbb{C P}^{n}$ in the case $n=2^{r}+1$ with $r \geq 1$. The information is taken from $[6 ; 14]$ in the case of $\mathrm{P}^{2 n+1}$, from $[15 ; 25]$ in the case of the immersion dimension of $L^{2 n+1}\left(2^{e}\right)$ for $e \geq 2$, from [14, Corollary 2] in the case of TC( $\left(\mathrm{PP}^{n}\right)$ and from $[3 ; 21]$ in the case of the immersion dimension of $\mathbb{C P}^{n}$. Note that in the case under consideration $\operatorname{TC}\left(\mathbb{C} \mathrm{P}^{n}\right)$ is just half the stable value of $\operatorname{TC}\left(L^{2 n+1}\left(2^{e}\right)\right)$ (ie, for $e \geq 3)$. Such a behavior comes from the fact that $\mathbb{C P}^{n}$ is simply connected and from Schwarz's estimates [24, Theorem 5] for the genus of a fibration.

\begin{tabular}{|c|c|c|c|c|}
\hline & $\mathrm{P}^{2 n+1}$ & $L^{2 n+1}(4)$ & $L^{2 n+1}\left(2^{e}\right) e \geq 3$ & $\mathbb{C P}^{n}$ \\
\hline TC & $\begin{array}{r}4 n-3(r \geq 2) \\
4 n-4(r=1)\end{array}$ & $4 n$ & $4 n+2$ & $2 n+1$ \\
\hline Imm & $4 n-4$ & $4 n-3$ & $4 n-2$ & $4 n-3$ \\
\hline
\end{tabular}

Table 2: TC vs. Imm for $2^{e}$-torsion lens spaces $\left(n=2^{r}+1, r \geq 1\right)$ 
We close this section by proposing what we believe should be an accessible challenge: Determine the symmetric topological complexity of high-torsion lens spaces. We remark that, in the high-torsion range, the inequalities

$$
4 n+2 \leq \mathrm{TC}^{S}\left(L^{2 n+1}(m)\right) \leq 4 n+3
$$

follow from (21), Theorem 5.7 and the analogue for lens spaces of the first inequality in (9).

\section{Complex projective spaces}

The (nonsymmetric) topological complexity of the $n$-dimensional complex projective space was computed in $[14$, Section 3$]$ to be $\operatorname{TC}\left(\mathbb{C P}^{n}\right)=2 n+1$. In this brief final section we show that the same value holds in the symmetric case.

Theorem 6.1 $\mathrm{TC}^{S}\left(\mathbb{C P}^{n}\right)=2 n+1$.

Proof In view of the analogue for complex projective spaces of the first inequality in (9), we only need to show that $\mathrm{TC}^{S}\left(\mathbb{C} \mathrm{P}^{n}\right) \leq 2 n+1$. The diagram of pullback squares

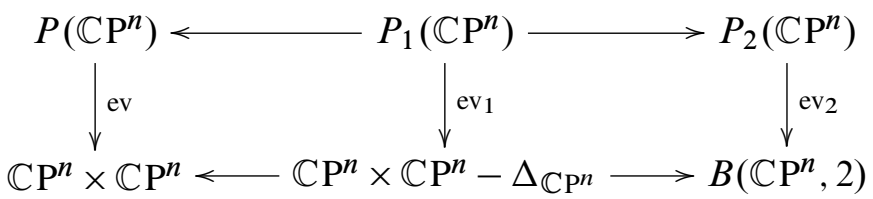

where horizontal maps on the left are inclusions, and horizontal maps on the right are canonical projections onto $\mathbb{Z} / 2$-orbit spaces, shows that the common fiber for the three vertical maps is the path connected space $\Omega \mathbb{C P}^{n}$. In particular, Theorem $5^{\prime}$ in [24] applied to $\mathrm{ev}_{2}$ gives

$$
\mathrm{TC}^{S}\left(\mathbb{C P}^{n}\right)=\operatorname{genus}\left(\mathrm{ev}_{2}\right)+1 \leq \frac{\operatorname{dim}(Y)}{2}+2
$$

where $Y$ is any $\mathrm{CW}$ complex having the homotopy type of $B\left(\mathbb{C P}^{n}, 2\right)$. The required inequality follows since, as indicated below, there is such a model $Y$ having $\operatorname{dim}(Y)=$ $4 n-2$.

In the proof of [12, Proposition 10] it is observed that, for a smooth closed $m-$ dimensional manifold $M, B(M, 2)$ has the homotopy type of a $(2 m-1)$-dimensional $\mathrm{CW}$ complex. Although this is certainly enough for completing the proof of Theorem 6.1 , we point out that an explicit (and smaller) model for $B\left(\mathbb{C P}^{n}, 2\right)$ was described by Yasui in [27, Proposition 1.6]. We recall the details. The unitary group $\mathrm{U}(2)$ has the two subgroups: 
$T^{2}$ : diagonal matrices

$G$ : matrices in $T^{2}$ together with those of the form $\left(\begin{array}{cc}0 & z_{1} \\ z_{2} & 0\end{array}\right)$ for $z_{1}, z_{2} \in S^{1}$.

Consider the standard action of $\mathrm{U}(2)$ on the complex Stiefel manifold $W_{n+1,2}$ of orthonormal 2-frames in $\mathbb{C}^{n+1}$ with quotient the Grassmann manifold of complex 2 -planes in $\mathbb{C}^{n+1}$. Yasui's model for $B\left(\mathbb{C P}^{n}, 2\right)$ is the corresponding quotient $W_{n+1,2} / G$. Note that $\operatorname{dim}(G)=\operatorname{dim}\left(T^{2}\right)=2$, so that the dimension of Yasui's model is

$$
\operatorname{dim}\left(W_{n+1,2}\right)-2=4 n-2 .
$$

\section{References}

[1] J Adem, S Gitler, I M James, On axial maps of a certain type, Bol. Soc. Mat. Mexicana (2) 17 (1972) 59-62 MR0336757

[2] L Astey, D M Davis, J González, Generalized axial maps and Euclidean immersions of lens spaces, Bol. Soc. Mat. Mexicana (3) 9 (2003) 151-163 MR1988595

[3] M F Atiyah, F Hirzebruch, Quelques théorèmes de non-plongement pour les variétés différentiables, Bull. Soc. Math. France 87 (1959) 383-396 MR0114231

[4] A J Berrick, S Feder, S Gitler, Symmetric axial maps and embeddings of projective spaces, Bol. Soc. Mat. Mexicana (2) 21 (1976) 39-41 MR0646069

[5] Z D Dai, T Y Lam, Levels in algebra and topology, Comment. Math. Helv. 59 (1984) 376-424 MR761805

[6] D M Davis, Table of immersions and embeddings of real projective spaces Available at http://www.lehigh.edu/ dmd1/immtable

[7] D M Davis, A strong nonimmersion theorem for real projective spaces, Ann. of Math. (2) 120 (1984) 517-528 MR769162

[8] M Farber, Topological complexity of motion planning, Discrete Comput. Geom. 29 (2003) 211-221 MR1957228

[9] M Farber, Instabilities of robot motion, Topology Appl. 140 (2004) 245-266 MR2074919

[10] M Farber, Topology of robot motion planning, from: "Morse theoretic methods in nonlinear analysis and in symplectic topology", (P Biran, O Cornea, F Lalonde, editors), NATO Sci. Ser. II Math. Phys. Chem. 217, Springer, Dordrecht (2006) 185-230 MR2276952

[11] M Farber, Invitation to topological robotics, Zurich Lect. in Adv. Math., Eur. Math. Soc., Zürich (2008) MR2455573 
[12] M Farber, M Grant, Symmetric motion planning, from: “Topology and robotics”, (M Farber, R Ghrist, M Burger, D Koditschek, editors), Contemp. Math. 438, Amer. Math. Soc. (2007) 85-104 MR2359031

[13] M Farber, M Grant, Robot motion planning, weights of cohomology classes, and cohomology operations, Proc. Amer. Math. Soc. 136 (2008) 3339-3349 MR2407101

[14] M Farber, S Tabachnikov, S Yuzvinsky, Topological robotics: motion planning in projective spaces, Int. Math. Res. Not. (2003) 1853-1870 MR1988783

[15] J González, Connective $K$-theoretic Euler classes and non-immersions of $2^{k}$-lens spaces, J. London Math. Soc. (2) 63 (2001) 247-256 MR1802770

[16] J González, Topological robotics in lens spaces, Math. Proc. Cambridge Philos. Soc. 139 (2005) 469-485 MR2177172

[17] A Haefliger, Plongements différentiables dans le domaine stable, Comment. Math. Helv. 37 (1962) 155-176 MR0157391

[18] A Haefliger, M W Hirsch, Immersions in the stable range, Ann. of Math. (2) 75 (1962) 231-241 MR0143224

[19] H Hopf, Systeme symmetrischer Bilinearformen und euklidische Modelle der projektiven Räume, Vierteljschr. Naturforsch. Ges. Zürich 85 (1940) 165-177 MR0004112

[20] W-C Hsiang, R H Szczarba, On the embeddability and nonembeddability of certain parallelizable manifolds, Bull. Amer. Math. Soc. 69 (1963) 534-536 MR0153024

[21] A Mukherjee, Embedding complex projective spaces in Euclidean space, Bull. London Math. Soc. 13 (1981) 323-324 MR620045

[22] E Rees, Embedding odd torsion manifolds, Bull. London Math. Soc. 3 (1971) 356-362 MR0296964

[23] E Rees, Embeddings of real projective spaces, Topology 10 (1971) 309-312 MR0288778

[24] A S Schwarz, The genus of a fiber space, Amer. Math. Soc. Transl., Ser. 255 (1966) 49-140

[25] T A Shimkus, Some new immersions and nonimmersions of $2^{r}$-torsion lens spaces, Bol. Soc. Mat. Mexicana (3) 9 (2003) 339-357 MR2029281

[26] B Steer, On the embedding of projective spaces in Euclidean space, Proc. London Math. Soc. (3) 21 (1970) 489-501 MR0286095

[27] T Yasui, The reduced symmetric product of a complex projective space and the embedding problem, Hiroshima Math. J. 1 (1971) 27-40 MR0314072 
Departamento de Matemáticas, CINVESTAV-IPN

AP 14-740 México City 07000, Mexico

Department of Mathematics, Rutgers University

110 Frelinghuysen Rd, Piscataway, NJ 08854-8019, USA

jesus@math.cinvestav.mx, landwebe@math.rutgers.edu

Received: 15 January 2009 Revised: 18 February 2009 